\title{
The Effects of Combination of Mimic miR-155-5p and Antagonist miR-324-5p Encapsulated Chitosan in Ovarian Cancer SKOV3
}

\author{
Rahma B Suardi ${ }^{1}$, Ysrafil Ysrafil ${ }^{2}$, Salsabila L Sesotyosari ${ }^{2}$, Ronny Martien ${ }^{3}$,Tirta \\ Wardana $^{4}$, Indwiani Astuti², Sofia M Haryana ${ }^{2 *}$
}

\begin{abstract}
Objective: Ovarian cancer is a malignant tumor that attacks reproductive organs of women. MicroRNA is known to have an involvement in the prognosis of ovarian cancer. One of them is miR-155-5p which is down regulated and miR-324-5p which is up regulated. Chitosan is used as microRNA delivery system. The aims of this study is to find out the effects of combination microRNA encapsulated chitosan in cell line SKOV3. Methods: Cell line SKOV3 obtained from Stem Cell and Cancer Institute (Kalbe). Mimic miR-155-5p and Antagonist miR-324-5p formulated with chitosan. Total RNA was extracted from nine samples (three as control and six as treatment), and prepared for cDNA synthesis. Expression of RNA and mRNA target was measured using q-PCR Biorad CFX96 C.100 and Gen Ex 7 software. Statistics analysis was measured using SPSS 16.0. Results: The administration of combination microRNA encapsulated with chitosan affect the expression of $m i R-155-5 p$ and $m i R-324-5 p$ endogen $(\mathrm{P}<0.05)$. The expression of mRNA target HIF1 $\alpha$ and GLI1 was down regulated after treatment. The correlation between expression of microRNA and mRNA target was strongly $(\mathrm{P}<0.05)$. Conclusion: This study successfully presented effects of combination of mimic miR-155-5p and antagonist miR-324-5p encapsulated chitosan which be considered as a potential therapy targets for ovarium cancer.
\end{abstract}

Keywords: Mimic miR-155-5p- antagonist miR-324-5p- chitosan- cell line SKOV3

Asian Pac J Cancer Prev, 21 (9), 2603-2608

\section{Introduction}

Ovarian cancer is one of the three main types of malignant tumors among all female gynecological tumors. Ovarian cancer exhibits no specific clinical symptoms in the early stage, resulting in the majority of patients coming in a state of cancer cells metastasis at the time of diagnosis (Li et al., 2016). The latest report indicate that the five-year survival rate of ovarian cancer is $42.9 \%$. However, more than $80 \%$ of patients with advanced ovarian cancer will relapse with a very poor prognosis. The extremely poor prognosis of ovarian cancer is known to be related to abnormal microRNA expression (Chen et al., 2019).

Micro RNAs (miRNAs) were discovered to be the endogenous non-coding small RNA molecules consisting of around 22 nucleotides. After transcription, the miRNAs regulate translation and expression of the mRNA target genes (Ferretti et al., 2008). Chasanah et al., (2016) found that expression of $h s a-m i R-155-5 p$ had decreased in the blood plasma of ovarian cancer. Hsa-miR-155-5p is a group of miRNA suppressor tumors that can bind and supress HIF $1 \alpha$ expression. HIF $1 \alpha$ is the mRNA target of miR-155-5p which functions as defense mechanism cancer cells in Hypoxia. Sumadi et al., (2018) also found that expression of $m i R-324-5 p$ increased in cell line SKOV3. Mir-324-5p is oncogenic and suppress tumor suppressor gene expression. Based on in silico research by Nurasih et al., (2018) miR-324-5p targeting GLI1 through Hedgehog signaling pathway.

Miroshnichenko et al., (2019) demonstrated the success of miRNA-based therapy combined with chemotherapy drugs to inhibit tumor development. Recent studies have shown a considerable increase in the efficacy of anticancer therapy as a synergistic effect of the therapy being applied simultaneously. MicroRNA derived from the same or different miRNA family, a mixture of anti-miRNA oligonucleotides and cytostatic drugs, and a combination of synthetic miRNA, have a complex effect compared with single miRNA therapy.

The success of administration miRNA into cancer cells is determined by dependence of specific, efficient

${ }^{1}$ Study Program of Biotechnology, Graduate School, Universitas Gadjah Mada, Yogyakarta, Indonesia. ${ }^{2}$ Faculty of Medicine, Public Health, and Nursing, Universitas Gadjah Mada, Yogyakarta, Indonesia. ${ }^{3}$ Facultyof Pharmacy, Universitas Gadjah Mada, Yogyakarta, Indonesia. ${ }^{4}$ Universitas Jenderal Soedirman, Central Java, Indonesia. *For Correspondence: sofia.mubarika@gmail.com 
and safe delivery. Chemical modification of miRNA synthetic could reduce miRNA ability. The development of the miRNA delivery system is focused use of nanoparticles transfection vectors. Nanoparticles are colloidal particles that range 1-10 nm, and are formulated using biodegradable polymers. One of the cationic polymers that have been utilized as delivery system using nanoparticles is chitosan.

Chitosan is a biodegradable polysaccharide composed of two D-glucosamine and N-acetyl-D-glucosamine subunits which are bound together with $(1,4)$ glycosidic bonds. The cationic properties of chitosan in acidic conditions enable the formation of complexes with negatively charged RNA (Winarti et al., 2011; SantosCarballal et al., 2015). Deng et al (2014) using doxorubicin and miR-34a are encapsulated with acid hyaluronicchitosan nanoparticles are proven to provide a synergistic effect on tumor suppression. This study was focused on the in vitro evaluation of the plausible application of combination miR-155-5p and miR-324-5p with chitosan nanoparticles in SKOV3 cell.

\section{Materials and Methods}

\section{Materials}

Cell lines SKOV3 was purchased from Stem Cell and Cancer Institute Kalbe. Mimic miR-155-5p and antogonist miR-324-5p were purchased from Integrated DNA Technologies, USA. Chitosan medium moleculer weight was purchased from Sigma-Aldrich ${ }^{\circledR}$. Primer mix miR-155-5p dan miR-324-5p (Intergrated DNA Technology, USA), Primer mRNA HIF1 $\alpha$, GLI1 dan $\beta$-actin (Intergrated DNA Technology, USA), miRCURY RNA Isolation Kit Cell and Plants (Cat. No.300110, Exiqon), Universal cDNA synthesis kit II 8-64 rxns (Cat. No.203301, Exiqon), ExiLent SYBR Green master mix, $2.5 \mathrm{ml}$ (Cat. No.203402, Exiqon), SensiFAST ${ }^{\mathrm{TM}}$ SYBR ${ }^{\circledR}$ (Cat. No. BIO-98020, Bioline).

Cell culture and chitosan microRNA formulation

The SKOV3 cell was cultured in DMEM culture medium containing 10\% fetal bovine serum (FBS) and placed in $5 \% \mathrm{CO}_{2}$ incubator chamber at $37^{\circ} \mathrm{C}$. Experimental groups were divided into control and combination miRNA transfected groups. Chitosan nanoparticles were prepared following the ionic gelation method using tripholyphospate (TPP) as cross linker. The chitosan-TPP transfection reagent, miR-155-5p mimic and antagonist miR-324-5p were mixed in a ratio $1: 1$ by volume, incubated in serum-free medium for 20 minutes.

\section{Characterization of nanoparticles chitosan}

Nanoparticles microRNA complex determined using electrophoresis according to protocol. The morphology of nanoparticles was evaluated using a transmission electron microscope (JEM-1400 JEOL). Samples were analyzed at $120 \mathrm{kV}$. A drop of the sample was deposited on a copper screen coated with carbon. The sample was dried and then contrasted with uranyl acetate for $2 \mathrm{~min}$ and then washed with distilled water.
In vitro cytotoxic activity of nanoparticles

The cultured cells were plated in 96-well culture dishes $\left(6 \times 10^{3}\right.$ cells), incubated for $24 \mathrm{~h}$ in a humidified atmosphere of $5 \% \mathrm{CO}_{2}$ and then treated with different concentrations of empty or combination miRNA loaded nanoparticles, incubation for $24 \mathrm{~h}$. A quantity of $100 \mu \mathrm{l}$ MTT was added in each well at four hours before testing. Formazan crystal dissolved in $100 \mu \mathrm{l}$ SDS were added to each well. After overnight incubation, the absorbency was measured by ELISA reader.

Oligonucleotide transfected into SKOV3 cell in vitro

SKOV3 cell of each group with concentration of $7 \times 10^{5} / \mathrm{ml}$ were seed in six well plates and incubated at $37^{\circ} \mathrm{C}$, in $5 \% \mathrm{CO}_{2}$ incubator for $24 \mathrm{~h}$. Then added chitosan nanoparticles combination microRNA to the wells and cultured. After 24 hours, RNA was isolated.

\section{$R N A$ isolation and quantification}

Total RNA extraction was isolated using miRCURY RNA Isolation Kit Cell and Plants, in compliance with the manufacturer's intructions. The total RNA from each samples were quantified using nanodrop to determine the RNA purity. The cDNA libraries were generated using Universal cDNA synthesis kit II 8-64 rxns. The quantification of expression miR-155-5p and miR-324-5p using ExiLent SYBR Green master mix, 2.5 $\mathrm{ml}$. The quantification of expression $m R N A H I F 1 \alpha$ and GLI1 using SensiFAST ${ }^{\mathrm{TM}} \mathrm{SYBR}^{\circledR}$. The quantification of expression of miRNA and $m R N A$ using quantitative PCR Biorad CFX96 C.100 (Bio-Rad ${ }^{\circledR}$ ). The expression was analyzed using Biorad CFX Manager ${ }^{\mathrm{TM}}$ Software and GenEx 7.

\section{Statistical analysis}

One-way ANOVA was used for the statistical analysis of the various experiments. A posteriori Bonferroni t-test was carried out to check the ANOVA test. A p value of $<0.05$ was considered statiscally significant.

\section{Results}

\section{Characterization of nanoparticles chitosan}

The ability of nanoparticles to interact and retain miRNA and the effective encapsulation were qualitatively investigated by agarose gel electrophoresis. Electrophoresis results showed that the microRNA combination complex without chitosan migrated from the well, while the chitosan-combination microRNA complex remained in the well (Figure 1). TEM analysis results the encapsulation of combination miR-155-5p and miR-324-5p with chitosan did not modify the morphology of the nanoplexes, which were characterized by a smooth spherical shape (Figure 2).

\section{In vitro cytotoxic activity of nanoparticles}

The in vitro antitumor effect of combination miRNA-loaded chitosan in terms of dose-response was investigated by using the MTT-test on SKOV3. The extremely low toxicity of empty chitosan towards SKOV3 cells was demonstrated, which means that any anti-proliferative effect that occurs has to be entrapped 


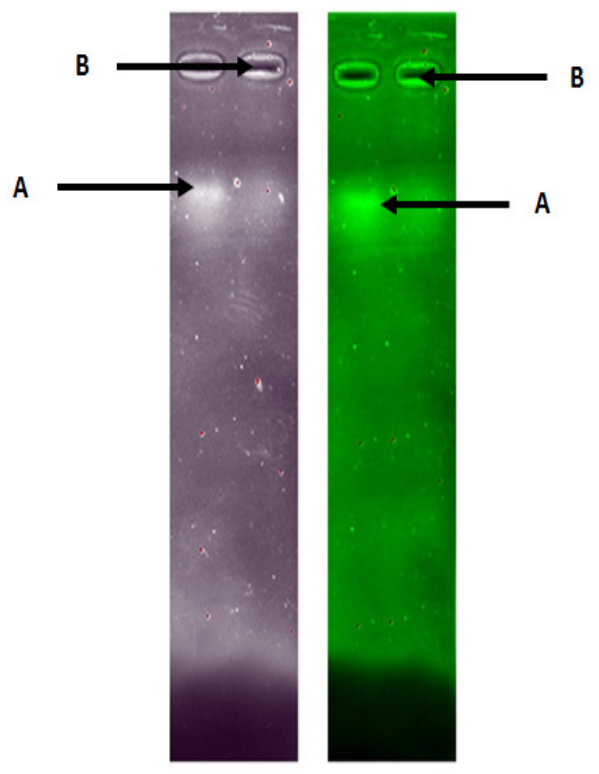

Figure 1. Chitosan-microRNA Nanoparticles were Run at $50 \mathrm{~V}$ for 20 Minutes. Note: (A) combination of naked microRNA, (B) combination of microRNA encapsulated chitosan

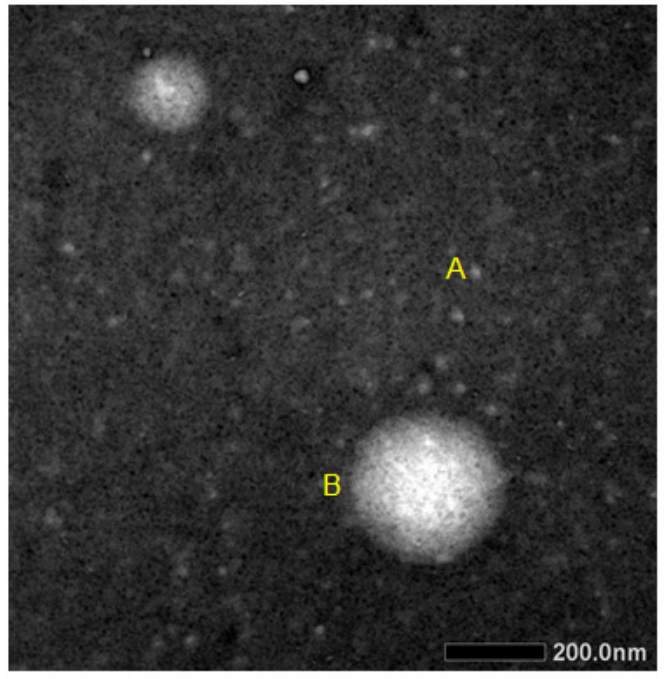

Figure 2. The Morphology of the Nanoparticles was Evaluated Using TEM. Samples were analyzed at $120 \mathrm{kV}$. TEM micrographs of empty nanoparticles/microRNA (A) and of nanoparticles/microRNA (B).

miRNA. Then it was observed that a significant decrease in cell viability was brought about by the chitosan nanoparticles at the highest combination miRNA (100 $\mathrm{nm})$ after $24 \mathrm{~h}$ incubation. In particular, at a combination miRNA concentration of $100 \mathrm{~nm}$, the nanoplexes induced

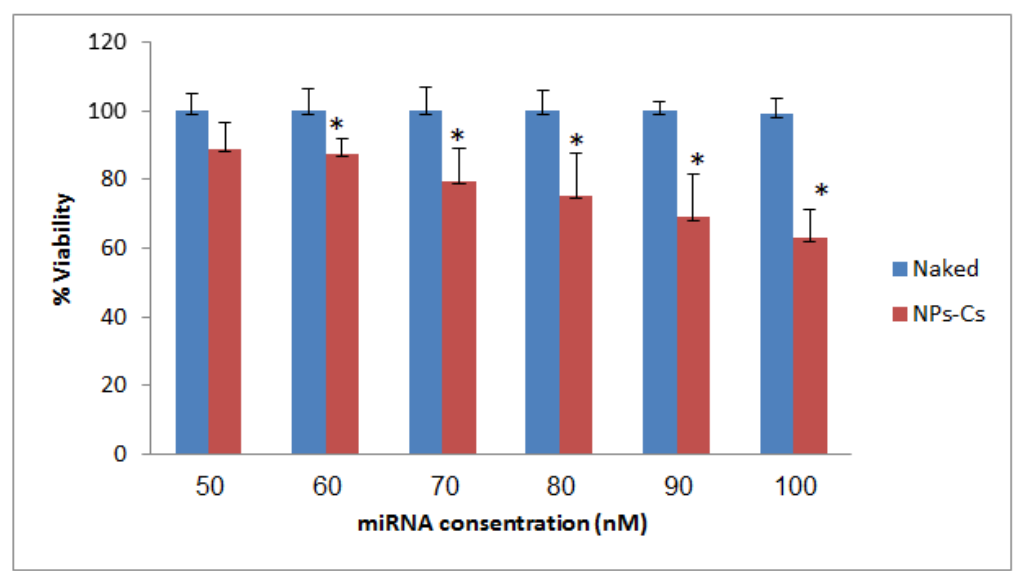

Figure 3. Percentage of inhibition of cell viability treated with chitosan-miRNA combination nanoparticles compared to combination treatment with miRNA without chitosan. Data are reported as the average mean \pm standart deviation. $* \mathrm{P}<0.05$. Naked: miRNA combination without chitosan; NPs-Cs: chitosan nanoparticles-miRNA combination
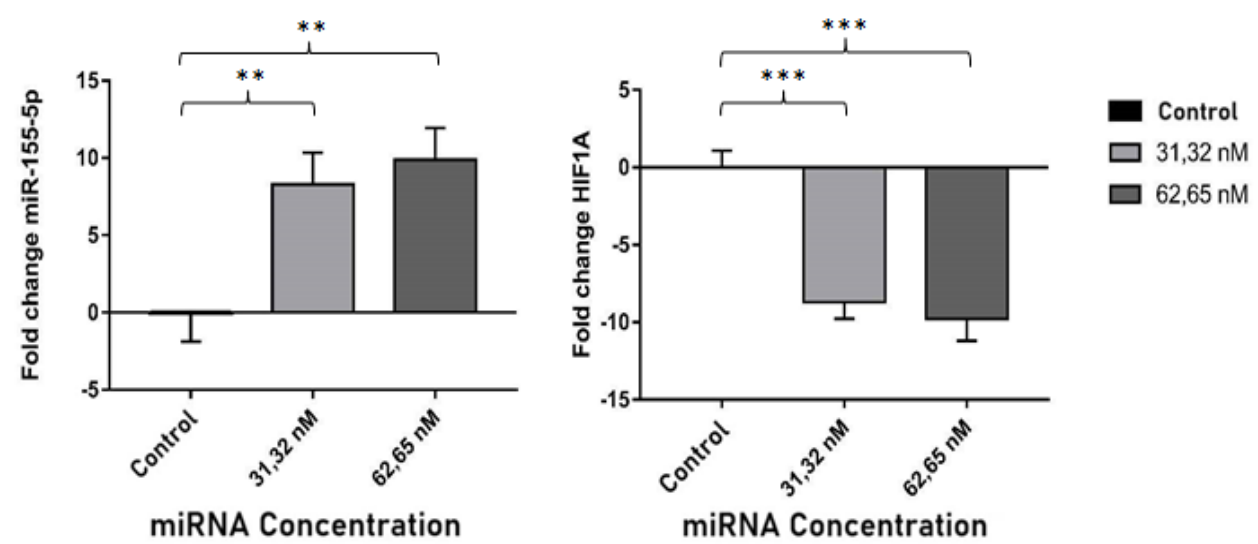

Figure 4. Fold Change of Endogenous miRNA-155-5p and HIF1 $\alpha$ mRNA Expression after Tranfected NPs-CS Combination of miRNA $31.32 \mathrm{nM}$ and $62.65 \mathrm{nM}$ Compared to Control Group. Data are presented in mean \pm SD. ** $\mathrm{P}<0.01$; *** $\mathrm{P}<0.001$. 

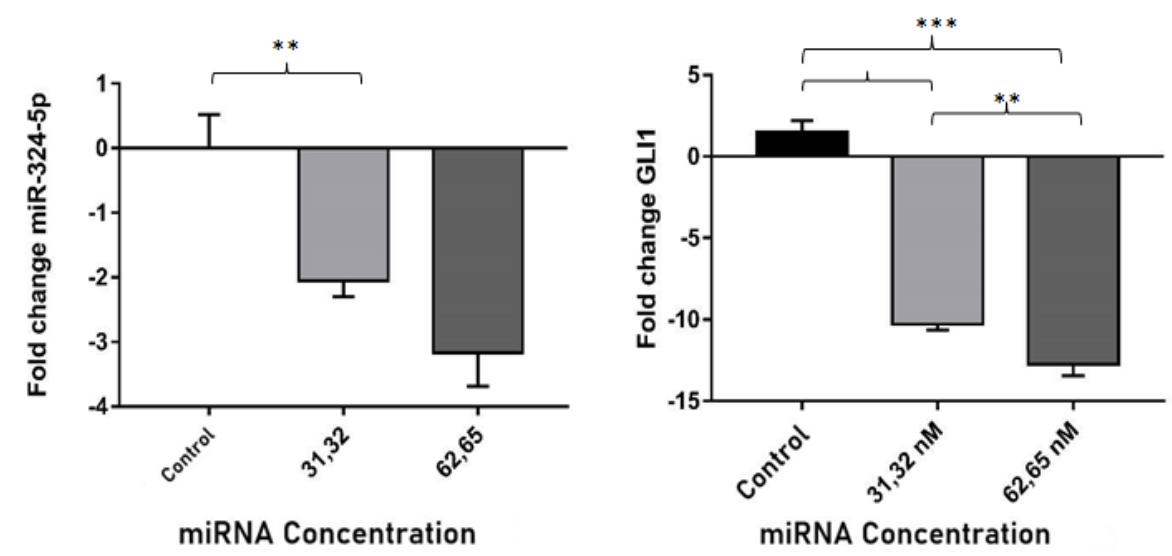

Figure 5. Fold change of endogenous miRNA-324-5p and mRNA GLI1 expression after transfected NPs-CS combination of miRNA $31.32 \mathrm{nM}$ and $62.65 \mathrm{nM}$ compared to the control group. Data are presented in mean $\pm \mathrm{SD}$. $* * \mathrm{P}<0.01 ; * * * \mathrm{P}<0.001$.

a decrease in cell viability of $40 \%$ on SKOV3 cells (Figure 3). There was significant difference between the transfected chitosan-miRNA groups compared to naked miRNA $(\mathrm{P}<0.05)$.

\section{MiR-155-5p expression and its correlation with HIF $1 \alpha$}

The q-PCR results show that the miR-155-5p expression increased significantly compared with the control group after the chitosan-combination miRNAs were transfected in the SKOV3, in terms of both 31,32 $\mathrm{nm}$ and $62,65 \mathrm{~nm}$ concentrations $(\mathrm{P}<0.05)$ (Figure 4). The miR-155-5p binding sites were in the HIF $1 \alpha$ mRNA 3'UTR region as predicted. In order to verify that HIF1 $\alpha$ was the target gene of the $m i R-155-5 p$, we measured the relative expression of $H I F 1 \alpha$. The results showed that the HIF $1 \alpha$ expression decreased significantly along with increased expression of endogenous miR-155-5p. This results showed than miR-155-5p could directly target HIF1 $\alpha$. Statistical analysis Pearson was measured to find out the correlation between the expression $m i R-155-5 p$ and mRNA HIF1 $\alpha$. The results showed that $p$ and $r$ value respectively 0.000 and -0.930 , indicated both of data have a strong negative correlations $(\mathrm{P}<0.05)$.

\section{MiR-324-5p expression and its correlation with GLI1}

The q-PCR results show that the $m i R-324-5 p$ expression decreased significantly compared with the control group after the chitosan-combination miRNAs were transfected in the SKOV3, in terms of both 31,32 nm and $62,65 \mathrm{~nm}$ concentrations $(\mathrm{P}<0.05)$ (Fig.5). The miR$324-5 \mathrm{p}$ binding sites were in the GLI1 mRNA 3 'UTR region as predicted by Gene Card software. In order to verify that GLI1 was the target gene of the miR-324-5p, we measured the relative expression of GLII. The results showed that the $G L I$ expression decreased significantly along with decreased expression of endogenous $m i R-324-5 p$. This results showed than GLI1 could be targeted by miR-3245 p. Statistical analysis Spearman was measured to find out the correlation between the expression $m i R-324-5 p$ and mRNA GLI1. The results showed that $p$ value 0.020 $(\mathrm{P}<0.05)$ and Spearman value 0.750 , indicated the expression of both have a positive correlations.

\section{Discussion}

Chitosan nanoparticles is used as a carrier of microRNA due to a high positive charge and low toxicity. This result was due to the ability of nanoparticles to package the poly-anionic combination miR-155-5p and miR-324-5p by way of an electrostatic interaction which comes about between the negatively-charged phosphate residue of nucleic acid and the positively-charged aminoresidues of chitosan. Chitosan nanoparticles formulation was carried out by ionic gelation method. The ionic gelation method involves a cross linker between the polyelectrolyte and its multivalent ion pair. The formation of a cross linker will strengthen of the particles formed. The polymer pair used for ionic gelation in this study was sodium tripolyphosphate.

The ability of nanoparticles to preserve microRNA and the effectiveness of encapsulation was qualitatively measured using electrophoresis. The result showed that the formation of chitosan/TPP-microRNA has been marked by the absence of bands in the microRNA combination encapsulated chitosan. This result due to the size of encapsulated microRNA become heavier caused difficultness to run in the pores of the gel (Winarti et al., 2013). Morphological testing of chitosan nanoparticles-microRNA combinations in this study produced nanoparticles in spherical shape and did not overlapping. Spherical nanoparticles have a greater uptake than rod shapes (Chithrani et al., 2007; Nur Laili et al., 2014).

The 3-[4,5-dimethylthiazol-2-ly]-3,5-diphenyltetrazolium bromide (MTT) test was performed on the SKOV3 cell lines in order to investigate the cytotoxicity of the colloidal formulation. MicroRNA as an anti-cancer candidate must be tested for its cytotoxicity to determine its inhibitory effect on SKOV3 cancer cells. The principle of this method is the addition of MTT reagents dissolved in PBS. Collection of enzymes found in mitochondria and cytosols of living cells, or known as mitochondrial dehydrogenase, will cut the tetrazole ring from MTT and reduce it to form purple formazan crystals.

Cytotoxicity tested with several variants of combinations miR-155-5p mimic and anti-miR-324- 
$5 \mathrm{p}$, both for naked miRNA and chitosan-miRNA nanoparticles. The dosage variations used were $50 \mathrm{~nm}, 60$ $\mathrm{nm}, 70 \mathrm{~nm}, 80 \mathrm{~nm}, 90 \mathrm{~nm}$ and $100 \mathrm{~nm}$. The result based on the graphic (Figure 3), variants of $100 \mathrm{~nm}$ concentration in miRNA nanoparticles can reduce cell viability $\pm 40 \%$. This dose variation refers to the study of Xu et al., (2014) using mimic miR-324-5p which was transfected to U87 cells (human glioma) able to inhibit glioma cell proliferation by $60 \%$ with a variation of $100 \mathrm{~nm}$ dose. In other words, the higher the concentration of the miRNA combination used, the higher the average percentage of cell death.

MicroRNA works by attaching to its target mRNA and causing translational suppression or degradation of its target mRNA. Thus, the researchers also calculated the target mRNA expression level of miR-155-5p, which is HIF $1 \alpha$. Jiang et al., (2006) shows that HIF $1 \alpha$ is strongly expressed in ovarian cancer. The high expression of $H I F 1 \alpha$ can increase the expression of both protein and $V E G F$ mRNA, which results in tumor growth and angiogenesis. Many in silico studies using miRNA target prediction algorithms, such as miRanda, TargetScan, and PicTar state that miR-155-5p has proven to target HIF1 $\alpha$. Chasanah et al., (2016) showed that the expression of $m i R-155-5 p$ in the blood serum of advanced stage ovarian cancer patients is lower than that of early-stage ovarian cancer patients. Correlates with increased HIF $1 \alpha$ expression in patients with advanced stage ovarian cancer compared with patients with early-stage ovarian cancer.

The expression of endogenous $m i R-155-5 p$ increased after being treated with miRNA combination nanoparticles caused decreased HIF $1 \alpha$ expression in SKOV3 ovarian cancer cells. Mimic miR-155-5p is designed to resemble endogenous miR-155-5p, so that it can be recognized by the RISC complex and can carry out the function of endogenous $m i R-155-5 p$ in suppressing the expression of its target mRNA. Statistical tests also showed endogenous miR-155-5p negatively correlated with HIF $1 \alpha$, with a very strong correlation strength.

Hypoxia-inducible factor (HIF) is a transcription factor that regulates the expression of various genes to be able to adapt in hypoxic conditions. One of the key factors governing cellular hypoxia response is Hypoxia-inducible factor 1 . HIF 1 is a heterodimer consisting of HIF Subunits $1 \alpha$ and HIF-1 $\beta$. HIF- $1 \alpha$ activity is regulated by proteosomal degradation that is mediated everywhere under normoxia conditions. When cancer cells lack oxygen, HIF $1 \alpha$ is stabilized and translocated to the nucleus, where HIF $1 \alpha$ activates angiogenesis and anaerobic metabolism. This mechanism play a role in the survival of cancer cells. Decreased HIF $1 \alpha$ expression can reduce the viability of SKOV3 cancer cells.

MiR-324-5p has a role as oncomiR which suppresses the expression of tumor suppressor genes. The expression miRNA-324-5p was decreased in the treated group compared to the control group. Chen et al., (2015) using anti-miR-21, which successfully suppresses the expression of endogenous miR-21 that is overexpressed. Anti-miR-21 blocks angiogenesis in breast cancer by inactivating the AKT and MAPK pathways.

According to Thomson et al., (2013) it is necessary to examine the expression of the target mRNA from miRNA to ensure that the transfected miRNA enters the target cell and performs its function to suppress gene expression. Results of GLI1 expression analysis after the transfected of miRNA combination, decreased expression significantly compared to the control group. This fact is also supported by the results of correlation analysis between $m i R-324$ $5 p$ expression and GLI1 which has a positive correlation. Ciucci et al., (2013) study stated that the level of GLI1 expression was found to be significantly increased in ovarian cancer epithelial cells compared to normal ovarian tissue. Prove that the combination of miRNA encapsulated chitosan can reduce GLI1 expression.

The molecular mechanism of miR-324-5p in targeting GLI1 in ovarian cancer cells is probably through Hedgehog pathway. Mir-324-5p may play an important role in key gene regulators in the Hedgehog pathway. Hedgehog $(\mathrm{Hh})$ pathway is a signal transduction pathway which has the main function as regulating physiological processes. Hedgehog pathway has main components namely Hh ligand which consists of: Patched receptor protein (Ptch), Smoothened transmembrane protein (Smo), cytoplasmic protein (Fused kinase, Cos2, GSK3 beta, PKA, SuFu, GLI). GLI is a downstream regulator in Hedgehog signaling. GLI1 has a function as an activator of transcription factors. Hedgehog signaling is affected by binding of extracellular proteins to the Ptch receptor, Smo becomes overexpressed and causes activation of the GLI1 transcription factor. GLI1 induces several target genes that play a role in cell progression such as: Cyclin D1, c-Myc, and Bcl-2. Abnormal expression of GLI1 affects the progression of cancer cells. The normal expression of miR-155-5p after tranfected can suppress the expression of HIF $1 \alpha$. The decreasing expression of $m i R-324-5 p$ can suppress the expression of GLI1. This study successfully presented effects of combination of mimic miR-155-5p and antagonist miR-324-5p encapsulated chitosan which be considerated as a potential targets therapy for ovarium cancer.

\section{Acknowledgements}

This study had received financial support from Fundamental Research Grants, by Ministry of Research \& Technology and Higher Education, The Republic of Indonesia (KEMENRISTEK DIKTI). This research is part of an approved thesis and has never been published in any scientific journal. There is no conflict of interest. The ethical issue was approved by medical and health research ethics committee (MHREC) fakulty of medicine Gadjah Mada University (Number of registration Ref: KE/FK/0949/EC/2018). The authors contributed equally to this work.

\section{References}

Chasanah SN, Fitriawan AS, Pukan FK, Kartika AI, Haryana SM (2016). The expression of miRNA-155 and hypoxia inducible factor alpha (HIF $1 \alpha)$ mRNA in the early and advanced stages of ovarian cancer patients blood plasma. J Med Sci, 48, 444-8.

Chen Y, Gao DY, Huang L (2015). In vivo delivery of miRNAs 
for cancer therapy: Challenges and strategies. Adv Drug Deliv Rev, 81, 128-41.

Chen SN, Chang R, Lin LT, et al (2019). MicroRNA in ovarian cancer: biology, pathogenesis, and therapeutic opportunities. Int J Environ Res Public Health, 16, 1510.

Chithrani BD, Chan WC (2007). Elucidating the mechanism of cellular uptake and removal of protein-coated gold nanoparticles of different sizes and shapes. J Nano Lett, 6, 1542-50.

Ciucci A, De Stefano I, Vellone VG, et al (2013). Expression of the Glioma-Associated Oncogene Homolog 1 (Gli1) in advanced serous ovarian cancer is associated with unfavorable overall survival. PLoS One, 8, e60145.

Deng X, Cao M, Zhang J, et al (2014). Hyaluronic acid-chitosan nanoparticles for co-delivery of miR-34a and doxorubicin in therapy against triple negative breast cancer. Biomaterials, 35, 4333-44.

Ferretti E, De Smaele E, Miele E, et al (2008). Concerted microRNA control of hedgehog signalling in cerebellar neuronal progenitor and tumour cells. Embo J, 27, 2616-27.

Jiang H, Feng Y (2006). Hypoxia-inducible factor $1 \alpha$ (HIF-1 $\alpha$ ) correlated with tumor growth and apoptosis in ovarian cancer. Int J Gynecol Cancer, 16, 405-12.

Li YT, Lee WL, Tsui KH (2016). Endometrial thickness still presents a best reference to predict endometrial cancer. J Obstet Gynecol, 55, 148-49.

Miroshnichenko S, Patutina O (2019). Enhanced inhibition of tumorigenesis using combinations of miRNA-targeted therapeutics. Front Pharmacol, 10, 485-88.

Nurasih AD, Haryana SM, Astuti I (2018). Development and testing of miR-155-5p mimic therapy targets and miR-324$5 p$ antagonists in SCOV3 ovarian cancer cell cultures. In Results and Discussions. Gadjah Mada University Press. Yogyakarta, pp 40-45.

Sumadi FA, Haryana SM, Martien R (2018). Microrna-based chitosan nanoparticle formulation as molecular targeted therapy in ovarian cancer cell culture SKOV3. In Results and Discussions. Gadjah Mada University Press. Yogyakarta pp 70-74.

Santos-Carballal B , Aaldering LJ, Ritzefeld M, et al (2015). Physicochemical and biological characterizationof chitosanmicroRNA nanocomplexes for gene delivery to MCF-7 breast cancer cells. $J$ Sci, 5, 13560-67.

Thomson DW, Bracken CP, Szuber JM, Goodall GJ (2013). On measuring miRNAs after Transient Transfection Mimics or Antisense Inhibitors. PLoS One, 8, 111-7.

Winarti L, Martien R, Sismindari (2013). Potential use of short chain chitosan as carriers in gene delivery: In Vitro Evaluation. Stomatognatic (J. K. G Unej) Volume 10 Number 2, pp 93-8.

Xu HS, Zong HL, Shang M, et al (2014). MiR-324-5p inhibits proliferation of glioma by target regulation of GLI1. Eur Rev Med Pharmacol, 18, 828-32.

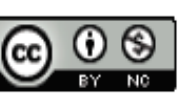

This work is licensed under a Creative Commons AttributionNon Commercial 4.0 International License. 